\title{
Vorsorgeprinzip beim Mobilfunk konsequent anwenden
}

\author{
Edith Steiner ${ }^{\mathrm{a}, \mathrm{d}}$, Bernhard Aufdereggen ${ }^{\mathrm{b}, \mathrm{d}}$, Cornelia Semadeni ${ }^{\mathrm{c}, \mathrm{d}}$ \\ ${ }^{a}$ Dr. med., Fachärztin für Innere Medizin; ${ }^{b}$ Dr. med., Facharzt für Allgemeine Innere Medizin; ${ }^{c}$ Fachärztin für Psychiatrie und Psychotherapie; \\ dArbeitsgruppe "Elektromagnetische Felder und Gesundheit» der Ärztinnen und Ärzte für Umweltschutz (AefU), Basel
}

Mobilfunkanbieter fordern aus wirtschaftlichen Gründen höhere Anlagegrenzwerte für ihre Mobilfunkanlagen. Schweizer Expertenberichte geben hinsichtlich gesundheitlicher Effekte der Mobilfunkstrahlung keine Entwarnung und fordern konsequente Vorsorge. Die Ärztinnen und Ärzte für Umweltschutz (AefU) verlangen, dass die ärztliche Expertise verstärkt in die digitale Agenda einbezogen wird.

Die Entwicklung des Mobilfunks macht uns Ärztinnen und Ärzten für Umweltschutz (AefU) Sorgen. Unsere "Arbeitsgruppe elektromagnetische Felder und Gesundheit» verfolgt seit Jahren die Studienlage zum Einfluss von nichtionisierender Strahlung (NIS) auf die Gesundheit. Wir pflegen Kontakte zu Forschenden und Behörden. Regelmässig sehen wir in unseren Praxen Menschen, die ihre Beschwerden auf den Einfluss von Mobilfunkstrahlung zurückführen. 2008 lancierten die AefU das Projekt «Umweltmedizinisches Beratungsnetz» mit dem Fernziel, umweltmedizinische Beratung als Bestandteil der Grundversorgung anzubieten. Unter dem Primat der Vorsorge fordern wir seit Anbeginn tiefere Grenzwerte, koordinierte Netzplanung, kontinuierliche For Information der Bevölkerung über die Ris gesundheitsverträglichere Nutzung von I Wir haben uns dazu bereits früher in der zerischen Ärztezeitung geäussert.

\section{Der Druck der Mobilfunkindust}

Im Zuge der Digitalisierung und der techr schen Möglichkeiten haben sich die Interessen der Mobilfunkanbieter auf die Ebene der Dienstleistungen verschoben. Mit schnellem Internet über Funk konkurrenzieren sie das bewährte und im Ausbau begriffene Festnetz (Glasfaser). Heute versorgen Mobilfunkantennen im Aussenraum hauptsächlich Nutzerinnen und Nutzer in Gebäuden. Sie verwenden 70 Prozent des Datenvolumens für
Videostreams. Mobilfunkantennen und Endgeräte verbrauchen den Löwenanteil ihrer Sendeleistung darauf, Mauern und Decken zu durchdringen. Das verursacht draussen und drinnen unnötige Strahlenbelastung.

\section{Die Entwicklung des Mobilfunks macht uns}

Ärztinnen und Ärzten für Umweltschutz Sorgen.

Der neue Mobilfunkstandard 5G soll seinen Anbietern neue Märkte erschliessen. Um billig noch mehr Geräte in Innenräumen von aussen mit Internet zu versorgen, fordern die Mobilfunkanbieter höhere Grenzwerte. Die so gewonnenen Kapazitäten wären schnell wieder ausgeschöpft. Wir Ärztinnen und Ärzte verlangen

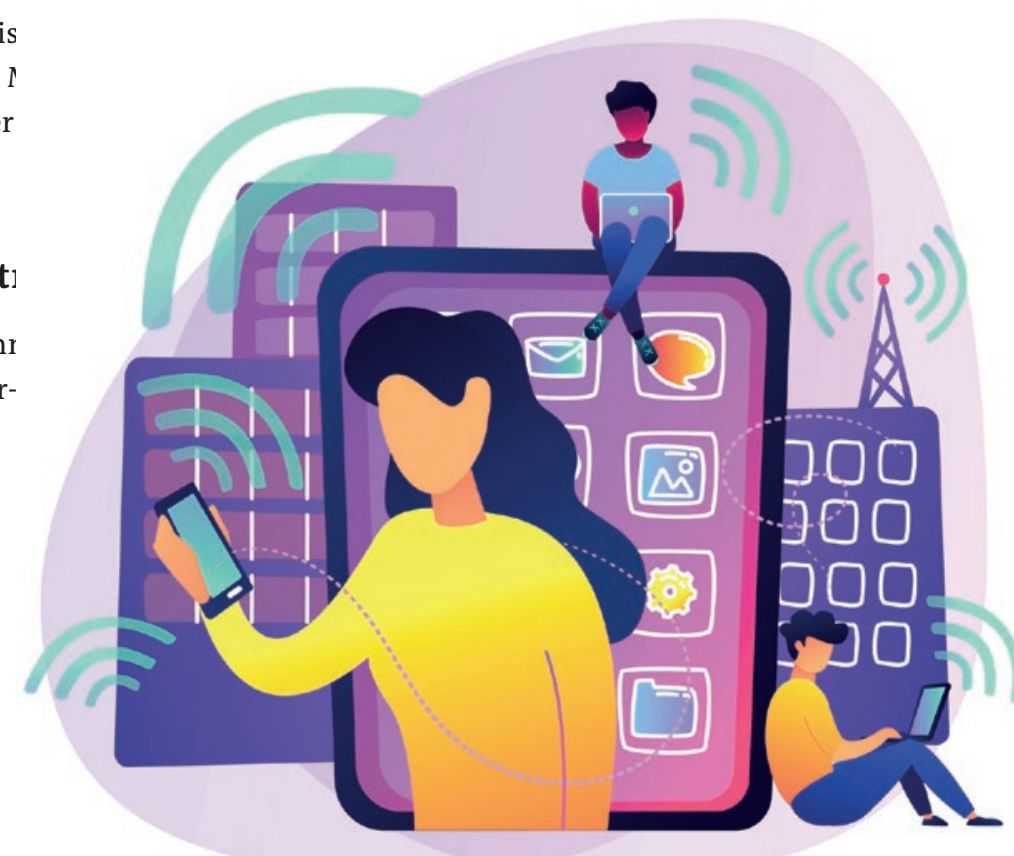


darum eine langfristig leistungsfähige Versorgung, getrennt nach Innen- und Aussenraum. Das schafft ein flächendeckendes Glasfasernetz bis in die Wohnungen, Schulen und zu den Arbeitsplätzen. Idealerweise haben die Endgeräte hier Kabelanschluss. Wo gewünscht, sind kabellose strahlenminimierte Kleinstnetzwerke zu installieren.

\section{Expertenberichte geben keine Entwarnung}

Hochfrequente Strahlung wurde von der WHO im Jahr 2011 als möglicherweise krebserregend für den Menschen eingestuft (Klasse 2B). Fall-Kontrollstudien zeigten ein erhöhtes Risiko für Gliome und vestibuläre Schwannome bei intensiver Handynutzung. Im Rahmen eines ersten politischen Vorstosses zur Lockerung der Mobilfunk-Grenzwerte beauftragte das Bundes-

\section{Das Seilziehen um die Grenzwerte geht weiter.}

amt für Umwelt BAFU 2014 ein interdisziplinäres Expertenteam. Es sollte eine aktuelle Bewertung der offenen Fragen betreffend die biologischen Effekte und gesundheitlichen Auswirkungen von schwacher Hochfrequenzstrahlung vornehmen. Der Bericht gab keine Entwarnung. Seither sichtet und beurteilt eine interdisziplinäre Expertengruppe im Auftrag des Bundes (BERENIS - Beratende Expertengruppe NIS) fortlaufend die Studienlage und publiziert einen regelmässigen Newsletter [1]. Die AefU sind in dieser Expertengruppe auch vertreten.

Zwischen 2014 und 2019 wurden drei methodisch als hochwertig qualifizierte Tierstudien publiziert. In einem etablierten Krebsmodell der Maus fördert Mobilfunkstrahlung das Tumorwachstum. In zwei unabhängig voneinander geführten Tierstudien zeigen männliche Ratten bei Lebendzeitexposition mit Mobilfunkstrahlung signifikant häufiger Schwannome am Herzen sowie trendweise Gliome am Hirn, also Tumoren der gleichen Zellart wie bei den Bevölkerungsuntersuchungen. Aufgrund der Ergebnisse dieser Tierstudien und neuer Hinweise für Effekte in Zellstudien empfahl das Beratungsgremium der internationalen Krebsagentur (IARC) im April 2019 eine Reevaluation des Krebsrisi$\operatorname{kos}[2]$.

\section{Bericht «Mobilfunk und Strahlung» als Diskussions-Basis}

Im Jahr 2019 beauftragte Altbundesrätin Doris Leuthard eine interdisziplinäre Arbeitsgruppe «Mobilfunk und Strahlung» mit der Aufgabe, Optionen aufzu- zeigen, wie unter Wahrung der Schutz- und Nutzinteressen das Mobilfunknetz ausgebaut werden kann, insbesondere im Hinblick auf die Einführung des neuen Mobilfunkstandard 5G. In der Arbeitsgruppe wirkten auch Vertreter der FMH und der AefU mit. Auch sie gab keine Entwarnung für die Effekte von Mobilfunkstrahlung und unterstützt das Vorsorgeprinzip [3]. Neben der offenen Frage zum Krebsrisiko können auch Auswirkungen auf Wohlbefinden, Elektrosensibilität, Kognition oder Fortpflanzung nicht eindeutig beurteilt werden. Zum als «Millimeterwellen» bezeichneten Frequenzbereich, der mittelfristig bei 5G eingesetzt werden soll, kann die Arbeitsgruppe keine robuste Risikobewertung machen. Es liegen zu wenig Studien vor.

Die Arbeitsgruppe konnte sich in ihrem im November 2019 publizierten Bericht auf keine gemeinsame Empfehlung einigen, wie Mobilfunknetze der Zukunft ausgestaltet sein sollen. Hingegen empfahl sie folgende Begleitmassnahmen: mehr Harmonisierung beim Vollzug, intensivere Forschung, Monitoring der Belastung und möglicher gesundheitlicher Auswirkungen, Information der Bevölkerung, die Schaffung einer umweltmedizinischen Beratungsstelle NIS (nichtionisierende Strahlung) sowie eine Austauschplattform.

\section{BERENIS fordert konsequente Anwendung des Vorsorgeprinzips}

Die internationale Strahlenschutzkommission (ICNIRP) hält in den im April 2020 erneuerten Richtlinien weiterhin an ihren Grenzwerten fest. Mit diesen Grenzwerten berücksichtigt sie nur wissenschaftlich konsistent nachgewiesene gesundheitliche Wirkungen auf den Menschen, nicht aber Hinweise für gesundheitliche Schädigung am Menschen sowie Ergebnisse von Tier- und Zellstudien.

Die BERENIS hat in ihrem Newsletter (Sonderausgabe)

\section{In einem etablierten Krebsmodell der Maus} fördert Mobilfunkstrahlung das Tumorwachstum.

vom Juli 2020 Stellung genommen zu den Richtlinien der ICNIRP. Sie stellt Unsicherheiten fest: Unterhalb der Grenzwerte der ICNIRP beeinflusst Mobilfunkstrahlung ausreichend evident die elektrische Aktivität des Gehirns und zeigt in Zell- und Tierstudien relativ konsistent Einflüsse auf oxidativen Stress. Die epidemiologische Studienlage zu Langzeitauswirkungen von Ganzkörperexpositionen über 1V/m ist unzureichend. Die BERENIS empfiehlt weiterhin die konsequente Anwendung des Vorsorgeprinzips. Dies gilt nicht nur für die Strahlung von fest installierten Sendeanlagen (An- 
tennen), auch die Minimierung der Exposition bei körpernah genutzten mobilen Endgeräten wird empfohlen.

\section{Das Seilziehen um die Vorsorge geht weiter}

Entgegen dem imperativen Wunsch der Mobilfunkanbieter und vieler Politikerinnen und Politiker hat der Bundesrat im April 2020 entschieden[4], den Anlagegrenzwert nicht zu erhöhen und die Begleitmassnahmen gemäss Bericht «Mobilfunk und Strahlung» umzusetzen. Das Eidgenössische Umweltdepartement UVEK erhielt den Auftrag, einen Bericht zu erstellen,

\section{Nicht nur die wirtschaftlichen Chancen, sondern auch die gesundheitlichen Risiken gehören auf die digitale Agenda.}

wie Mobilfunknetze nachhaltig ausgestaltet werden können. Doch das Seilziehen um die Grenzwerte geht weiter. Die Mobilfunk-Betreiber umwerben mit ihrer neuen Plattform "Chance5G» Politik, Wirtschaft und Bildung mit ihrem Anliegen. Wir von den AefU haben die vorsorgebasierten Forderungen zu Mobilfunk und 5G in einem aktuellen Positionspapier [5] und in unserer Fachzeitschrift OEKOSKOP 2/2020 [6] festgehalten.

\section{Gesundheitliche Risiken gehören auf die digitale Agenda}

Mobilfunk durchdringt und dominiert unseren Alltag. Das erhöht nicht nur die NIS-Belastung (niederfrequente und hochfrequente elektromagnetische Felder/Blaulicht), sondern führt auch zu einer massiven Veränderung der Lebensgewohnheiten und des Verhaltens mit entsprechendem relevanten Gesundheitsrisiko (Suchtverhalten, Schlafstörungen, Depression, Haltungsschäden, Kurzsichtigkeit, Entwicklungsstörungen) [7]. Dies betrifft insbesondere Kinder und
Jugendliche. Sie nutzen diese Technologien häufig und lange in ihrem Alltag und beginnen damit auch immer früher. Sie sind besonders sensibel für Ausseneinflüsse, weil ihr Körper und Geist sich noch in Entwicklung befinden. Gerade für diese Generation ist es wichtig, dass sich die politische und gesellschaftliche Diskussion versachlicht. Die wissenschaftlich begründbaren Sorgen dürfen nicht als Angstmacherei, Technologiefeindlichkeit oder Unwissenheit abgetan werden. Der Bericht «Mobilfunk und Strahlung» bietet eine Basis für die politische und gesellschaftliche Debatte um den Mobilfunk der Zukunft. Es wäre wünschenswert, dass diese Auseinandersetzung mit der Digitalisierung fortan sachlich, faktenbasiert und lösungsorientiert stattfindet, auch im Rahmen der vorgeschlagenen Austauschplattform. Nicht nur die wirtschaftlichen Chancen, sondern auch die gesundheitlichen Risiken gehören auf die digitale Agenda. Dabei ist die ärztliche Expertise unverzichtbar.

\section{Bildnachweis}

(c) Visual Generation | Dreamstime.com

\section{Literatur}

1 Beratende Expertengruppe NIS (BERENIS): Newsletter https:// www.bafu.admin.ch/bafu/de/home/themen/elektrosmog/ newsletter.html

2 IARC Monographs Priorities Group. Advisory Group recommendations on priorities fort he IARC Monographs. Lancet Oncol. 2019; 20(6):763-4.

3 Arbeitsgruppe Mobilfunk und Strahlung. Bericht Mobilfunk und Strahlung. Im Auftrag des UVEK. 18. November 2019.

4 Bundesamt für Umwelt BAFU. «Bundesrat entscheidet über weiteres Vorgehen im Bereich Mobilfunk und 5G». Medienmitteilung vom 22.4.2020. https://www.bafu.admin.ch/bafu/de/home/ themen/elektrosmog/dossiers/bericht-arbeitsgruppe-mobilfunkund-strahlung.html

5 AefU-Position Mobilfunk und Strahlung, Juni 2020. http://aefu.ch/ fileadmin/user_upload/aefu-data/b_documents/themen/ elektrosmog/aefu_position-nis.pdf

6 http://www.aefu.ch/fileadmin/user_upload/aefu-data/b_documents/oekoskop/Oekoskop_20_2.pdf

7 BERENIS Newsletter 19, Seite 3. https://www.bafu.admin.ch/bafu/ de/home/themen/elektrosmog/newsletter.html
Ärztinnen und Ärzte für Umweltschutz (AefU) Arbeitsgruppe elektro magnetische Felder und Gesundheit Dr. med. Edith Steiner Postfach 620 CH-4019 Basel info[at]aefu.ch

\section{Das Wichtigste in Kürze}

- Die Entwicklung des Mobilfunks macht uns Ärztinnen und Ärzten für Umweltschutz (AefU) Sorgen.

- Wir fordern die konsequente Anwendung des Vorsorgeprinzips.

- Die politische und gesellschaftliche Diskussion rund um die Gefahren der elektromagnetischen Felder sowie des Blaulichts soll sich versachlichen.

- Die wissenschaftlich begründbaren Sorgen sollen nicht als Angstmacherei, Technologiefeindlichkeit oder Unwissenheit abgetan werden.

- Kinder und Jugendliche sind als besonders sensible Gruppe zu schützen, weil sie dieTechnologie häufig und lange im Alltag nutzen und auch immer früher damit beginnen.

\section{L'essentiel en bref}

- Nous, les Médecins en faveur de l'Environnement (MfE), nous inquiétons du développement de la téléphonie mobile.

- Nous demandons I'application rigoureuse du principe de précaution.

- Le débat politique et social sur les dangers des champs électromagnétiques et de la lumière bleue doit être objectivé.

- Les préoccupations scientifiquement justifiables ne doivent pas être décriées comme étant alarmistes, technophobes ou ignorantes.

- Les enfants et les adolescents doivent être protégés en tant que groupe particulièrement sensible, parce qu'ils utilisent souvent et longtemps cette technologie au quotidien et commencent de plus en plus jeunes. 